\title{
Correction to: Full genome sequence of a polyvalent bacteriophage infecting strains of Shigella, Salmonella, and Escherichia
}

\author{
Alaaeldin Mohamed Saad ${ }^{1,2} \cdot$ Ahmed Askora $^{3} \cdot$ Ahmed Mahrous Soliman $^{4} \cdot$ Hirofumi Nariya ${ }^{4} \cdot$ Takeru Kawasaki $^{1}$. \\ Makoto Fujie ${ }^{1}$ Tadashi Shimamoto ${ }^{4} \cdot$ Takashi Yamada $^{1,5}$ (D)
}

Published online: 22 August 2018

C) Springer-Verlag GmbH Austria, part of Springer Nature 2018

\section{Correction to: Archives of Virology https://doi.org/10.1007/s00705-018-3971-y}

The original version of this article unfortunately contained a mistake. Ahmed Mahrous Soliman, Hirofumi Nariya and Tadashi Shimamoto were not listed among the authors. The author group should be:

Alaaeldin Mohamed Saad ${ }^{1,2}$, Ahmed Askora ${ }^{3}$, Ahmed Mahrous Soliman ${ }^{4}$, Hirofumi Nariya ${ }^{4}$, Takeru Kawasaki ${ }^{1}$, Makoto Fujie $^{1}$, Tadashi Shimamoto ${ }^{4}$, Takashi Yamada ${ }^{1,5}$

${ }^{1}$ Department of Molecular Biotechnology, Graduate School of Advanced Sciences of Matter, Hiroshima University, Higashi-Hiroshima, 739-8530, Japan

The original article can be found online at https://doi.org/10.1007/ s00705-018-3971-y.

Takashi Yamada

tayamad@hiroshima-u.ac.jp

1 Department of Molecular Biotechnology, Graduate School of Advanced Sciences of Matter, Hiroshima University, Higashi-Hiroshima 739-8530, Japan

2 Department of Zoonoses, Faculty of Veterinary Medicine, Zagazig University, Zagazig, Egypt

3 Department of Microbiology, Faculty of Science, Zagazig University, Zagazig, Egypt

4 Laboratory of Food Microbiology and Hygiene, Graduate School of Biosphere Science, Hiroshima University, Higashi-Hiroshima 739-8528, Japan

5 Hiroshima Study Center, The Open University of Japan, Hiroshima 730-0053, Japan
${ }^{2}$ Department of Zoonoses, Faculty of Veterinary Medicine, Zagazig University, Zagazig, Egypt

${ }^{3}$ Department of Microbiology, Faculty of Science, Zagazig University, Zagazig, Egypt

${ }^{4}$ Laboratory of Food Microbiology and Hygiene, Graduate School of Biosphere Science, Hiroshima University, Higashi-Hiroshima, 739-8528, Japan

${ }^{5}$ Hiroshima Study Center, The Open University of Japan, Hiroshima, 730-0053, Japan 